\title{
An Inverse Sturm-Liouville Problem with a Fractional Derivative
}

\author{
Bangti Jin* William Rundell*
}

May 29, 2018

\begin{abstract}
In this paper, we numerically investigate an inverse problem of recovering the potential term in a fractional Sturm-Liouville problem from one spectrum. The qualitative behaviors of the eigenvalues and eigenfunctions are discussed, and numerical reconstructions of the potential with a Newton method from finite spectral data are presented. Surprisingly, it allows very satisfactory reconstructions for both smooth and discontinuous potentials, provided that the order $\alpha \in(1,2)$ of fractional derivative is sufficiently away from 2 .
\end{abstract}

\section{Introduction}

We consider the Sturm-Liouville problem (SLP) for the fractional differential equation

$$
\left\{\begin{array}{l}
-D_{0}^{\alpha} u+q u=\lambda u \quad 0<x<1, \\
u(0)=u(1)=0
\end{array}\right.
$$

where the (left-sided) Caputo fractional derivative $D_{0}^{\alpha}$ of order $\alpha \in(1,2)$ is defined by

$$
D_{0}^{\alpha} u(x)=\frac{1}{\Gamma(2-\alpha)} \int_{0}^{x} \frac{u^{\prime \prime}(t)}{(x-t)^{\alpha-1}} d t,
$$

where $\Gamma(\cdot)$ refers to the standard Gamma function. We shall normalize the eigenfunction $u$ by $u^{\prime}(0)=1$. As the exponent $\alpha$ tends to 2, problem (1) reduces to the classical SLP [10, Thm. 2.1, pp. 92]. The fractional SLP (1) is of immense interest for several reasons.

First, it arises naturally in the analysis of (spatially) fractional wave equations when applying Fourier transform. Fractional wave equations are often used to faithfully capture dynamical behaviors of amorphous materials, e.g., polymer and porous media; see the comprehensive reviews [17, 22] and references therein. Hence, a better understanding of the SLP (1) would shed valuable insight into the underlying physics of these phenological models.

Second, diffusion is one of the most prominent transport mechanisms found in nature. At a microscopic level, it is the result of the random motion of individual particles, and the use of the Laplace operator in the canonical model rests on a Gaussian process assumption on the random motion. Over the last twenty years a wide body of the literature has shown that anomalous diffusion in which the mean square variance grows faster (superdiffusion) or slower (subdiffusion) than in a Gaussian process offers a superior fit to experimental data (see the reviews [29, 26, 2, 14, 15]). This is particularly true in materials with memory, e.g., viscoelastic materials. This anomalous diffusion can be in either the spatial or temporal variables and is typically reflected in a fractional order derivative. In either case, separation of variables leads to a (fractional order) ordinary differential equation with a set of constants, i.e., the eigenvalues.

Third, it serves as a natural departure from the classical SLP $(\alpha=2)$, for which there is a wealth of profound theoretical results for both the forward and inverse problems (see [4, Chap. 3] for an overview).

\footnotetext{
*Department of Mathematics and Institute for Applied Mathematics and Computational Science, Texas A\&M University,
} College Station, Texas 77843-3368, USA (btjin,rundell@math.tamu.edu) 
It is clearly interesting to see how these results might be translated to the new scenario both from a mathematical perspective and for what this tells us about the underlying physics.

Suppose that we wish to solve the inverse problem of recovering the potential $q(x)$ from one spectrum $\left\{\lambda_{k}\right\}$. It is well known that for the classical SLP $(\alpha=2)$, this is in general insufficient. One spectrum completely determines the potential only if additional a priori information is available, for example, if it is known to be symmetric about the midpoint of the interval or is given on one half of the interval and has to be determined on the other half. In case of a general potential and fixed boundary conditions at the endpoints one requires additional information in the form of a second sequence. This can be a second spectrum arising from different boundary conditions, specifying both the eigenfunction and its derivative at an endpoint, or giving the energy associated with each frequency - the so-called norming constants (see [4, Chap. 3]). However there are many situations where this additional knowledge cannot be obtained, and one natural question is whether the same requirements also hold for the fractional case, (1).

A number of efficient reconstruction techniques, including an iterative method involving an associated hyperbolic equation [24], variational methods [21], and linearization methods using an iterated Newton method based on the coefficient-to-data map [13], have been developed for the classical case.

The main result of this paper is strong evidence that the fractional SLP (1) has very different properties and extensive numerical results lead to the conclusion that one spectrum completely determines a general potential $q$ for $1<\alpha<2$, which contrasts sharply with the classical case of $\alpha=2$. In this paper we will also deal with reconstruction methods and follow the Newton scheme as the method of choice.

We note that despite the extensive literature on the forward problem on fractional ordinary/partial differential equations (see $[8,19,10,11,3,25]$ for an incomplete list) and their diverse physical and engineering applications [16], the research on relevant inverse problems remains very scarce. Recently, several works on unique identifiability and numerical methods for inverse problems for time-fractional diffusion/wave equations $[5,12,31]$ have appeared and some unusual phenomena over the classical case has been observed.

The paper is structured as follows. In Section 2 we discuss results about the Sturm-Liouville theory for fractional differential operators. In particular, we develop an asymptotic formula for the spectrum and indicate the main properties of the eigenfunctions. It turns out that the spectral values are complex and so we have both a real and imaginary parts to the eigenfunctions and it is this feature that allows the identifiability from a single, albeit complex spectrum. In Section 3 we numerically investigate the inverse SLP with a Newton-type method. Numerical results for both smooth and discontinuous potentials clearly illustrate the phenomenon of recovering a general potential for (1) from one spectrum. We conclude the paper with some future research problems in Section 4.

\section{Sturm-Liouville theory}

In this section, we discuss qualitative behaviors of the spectrum to the SLP (1). We first discuss the case of a zero potential, which unlike the situation with $\alpha=2$, is quite nontrivial, and then turn to the case of a nonzero potential.

\subsection{Differential operator $-D_{0}^{\alpha}$}

In this part, we collect known results about the spectra of the fractional differential operators $-D_{0}^{\alpha}(1<$ $\alpha<2)$. We shall use the two-parameter Mittag-Leffler function $E_{\alpha, \beta}(z)$ defined by

$$
E_{\alpha, \beta}(z)=\sum_{k=0}^{\infty} \frac{z^{k}}{\Gamma(\beta+\alpha k)} .
$$

The two particular versions of Mittag-Leffler function relevant to problem (1) are $E_{\alpha, 2}(z)$ and $E_{\alpha, \alpha}(z)$. The former occurs in the form $x E_{\alpha, 2}\left(-\lambda x^{\alpha}\right)$ as the eigenfunctions of (1) with $q=0$, while the second arises in the kernel of the one-sided Green's function for (1) with $q=0$. These functions will also appear 
later in the Jacobian matrix coming from the linearized problem. Further, the Dirichlet eigenvalues of the operator $D_{0}^{\alpha}$ coincide with zeros of $E_{\alpha, 2}(z)$ [18, Thm. 4].

If $0<\alpha<2$ and $\mu \in\left(\frac{\alpha \pi}{2}, \min (\pi, \alpha \pi)\right)$, then the function $E_{\alpha, \beta}(z)$ has the following exponential asymptotic expansion (see e.g., [8, Thm. 1.3-4, pp. 5], [10, eq. (1.8.27), p. 43]): as $|z| \rightarrow \infty$

$$
E_{\alpha, \beta}(z)=\left\{\begin{array}{lr}
\frac{1}{\alpha} z^{\frac{1-\beta}{\alpha}} \exp \left(z^{\frac{1}{\alpha}}\right)-\sum_{k=1}^{N} \frac{1}{\Gamma(\beta-\alpha k)} \frac{1}{z^{k}}+O\left(\frac{1}{z^{N+1}}\right), & |\arg (z)| \leq \mu, \\
-\sum_{k=1}^{N} \frac{1}{\Gamma(\beta-\alpha k)} \frac{1}{z^{k}}+O\left(\frac{1}{z^{N+1}}\right), & \mu \leq|\arg (z)| \leq \pi .
\end{array}\right.
$$

The fractional SLP (1) was studied earlier [7, 18]. In [7], the existence of a solution to such boundary value problem was established, and a certain biorthogonal system was constructed from the eigenfunctions, see also [8, Chaps. 10-12]. In [18], the aforementioned relation between eigenvalues and zeros of Mittag-Leffler function was shown. The distribution of the zeros of the function $E_{\alpha, \beta}(z)$ is of independent interest [8, Chap. 1] [27, 20]. The next result [8, Lemma 1.4-2, pp. 7] represents one of the main results. For the sake of completeness, we sketch the proof.

Theorem 2.1. For all sufficiently large $n$ (in absolute value), the zeros $\left\{z_{n}\right\}$ of the Mittag-Leffler function $E_{\alpha, 2}(z)$ are simple, and have the following asymptotics

$$
z_{n}^{\frac{1}{\alpha}}=2 n \pi \mathrm{i}-(\alpha-1)\left(\ln 2 \pi|n|+\frac{\pi}{2} \operatorname{sign}(n) \mathrm{i}\right)+\ln \frac{\alpha}{\Gamma(2-\alpha)}+d_{n},
$$

where the remainder $d_{n}$ is $O\left(\frac{\ln |n|}{|n|}\right)$.

Proof. By taking $N=1$ in the exponential asymptotics (2), we get

$$
E_{\alpha, 2}(z)=\frac{1}{\alpha} z^{-\frac{1}{\alpha}} e^{z^{\frac{1}{\alpha}}}-\frac{1}{\Gamma(2-\alpha)} \frac{1}{z}+O\left(\frac{1}{z^{2}}\right)
$$

for $|z| \rightarrow \infty$. Hence we have

$$
z^{1-\frac{1}{\alpha}} e^{z^{\frac{1}{\alpha}}}=\frac{\alpha}{\Gamma(2-\alpha)}+O\left(\frac{1}{z}\right)
$$

Next let $\zeta=z^{\frac{1}{\alpha}}$ and $w=\zeta+(\alpha-1) \ln \zeta$. Then the equation can be rewritten as

$$
e^{w}=\frac{\alpha}{\Gamma(2-\alpha)}+O\left(\frac{1}{w^{\alpha}}\right) .
$$

The solutions $w_{n}$ to the above equation for all sufficiently large $n$ satisfy

$$
w_{n}=2 \pi n \mathrm{i}+\ln \frac{\alpha}{\Gamma(2-\alpha)}+O\left(\frac{1}{|n|^{\alpha}}\right)
$$

or equivalently

$$
\zeta_{n}+(\alpha-1) \ln \zeta_{n}=2 \pi n \mathrm{i}+\ln \frac{\alpha}{\Gamma(2-\alpha)}+O\left(\frac{1}{|n|^{\alpha}}\right),
$$

from which we arrive at the desired assertion

$$
\zeta_{n}=2 \pi n \mathrm{i}+\ln \frac{\alpha}{\Gamma(2-\alpha)}-(\alpha-1)\left(\ln 2 \pi|n|+\frac{\pi}{2} \operatorname{sign}(n) \mathrm{i}\right)+O\left(\frac{\ln |n|}{|n|}\right) .
$$


We can improve the estimate in Theorem 2.1 by including further terms in the residual tail in the asymptotic (2), $N \geq 2$, but with considerable increases in the complexity of the result.

Clearly, if $\lambda \in \mathbb{C}$ is an eigenvalue of $-D_{0}^{\alpha}$, then its conjugate $\bar{\lambda}$ is also an eigenvalue. Hence we shall restrict our discussions to $n>0$. As a direct consequence of Theorem 2.1, there are only finitely many real eigenvalues for any $\alpha<2$. The existence of real eigenvalues is only guaranteed for $\alpha$ sufficiently close to 2 [20]. Further, asymptotically, the eigenvalues $\left\{\lambda_{n}\right\}$ are distributed as

$$
\left(-\lambda_{n}\right)^{\frac{1}{\alpha}} \sim 2 \pi n \mathrm{i}+\ln \frac{\alpha}{\Gamma(2-\alpha)}-(\alpha-1)\left(\ln 2 \pi n+\frac{\pi}{2} \mathrm{i}\right) .
$$

Hence, we immediately get the following corollary.

Corollary 2.1. Asymptotically, the magnitude $\left|\lambda_{n}\right|$ and phase $\arg \left(\lambda_{n}\right)$ of the $n^{\text {th }}$ eigenvalue $\lambda_{n}$ are given by

$$
\begin{aligned}
\left|\lambda_{n}\right| & \sim\left(\left(2 \pi n+(1-\alpha) \frac{\pi}{2}\right)^{2}+\left((1-\alpha) \ln 2 \pi n+\ln \frac{\alpha}{\Gamma(2-\alpha)}\right)^{2}\right)^{\frac{\alpha}{2}} \sim(2 \pi n)^{\alpha}, \\
\arg \left(\lambda_{n}\right) & \sim \pi-\alpha \operatorname{atan} \frac{2 \pi n+(1-\alpha) \frac{\pi}{2}}{(\alpha-1) \ln 2 \pi n-\ln \frac{\alpha}{\Gamma(2-\alpha)}} \sim \frac{(2-\alpha) \pi}{2} .
\end{aligned}
$$

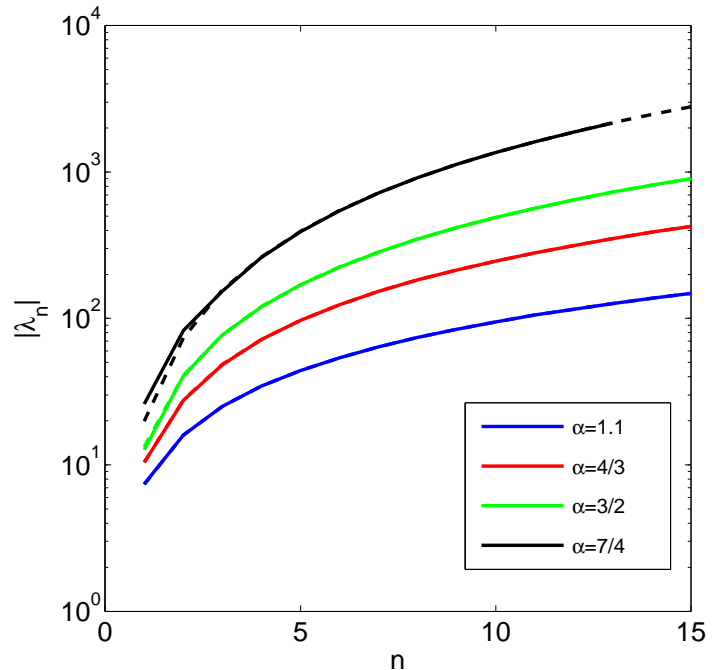

(a) magnitude $\left|\lambda_{n}\right|$

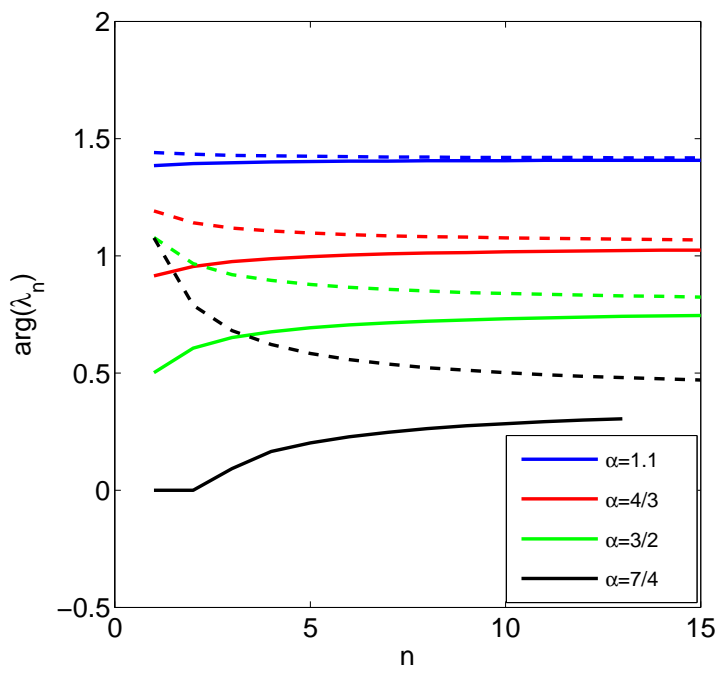

(b) phase $\arg \left(\lambda_{n}\right)$

Figure 1: Numerical verification of the asymptotic formula (3): the solid and dashed lines represent the true and predicted values, respectively, for $\alpha=1.1,4 / 3,3 / 2$ and $7 / 4$.

We show in Figure 1 the magnitude and phase of true eigenvalues and their predictions by the asymptotic formula (3). The true eigenvalues are calculated by a quasi-Newton method (cf. Appendix $\mathrm{B}$ ), and the values are precise in the first six digits. The magnitudes can be accurately predicted by formula (3), except the first few eigenvalues. Hence, it might allow extracting the exponent $\alpha$ directly from the sequence of eigenvalues. The approximation of the phase is not as good, cf. Figure 1(b), and it is accurate only for very large $n$, especially for $\alpha$ values close to 2 . This is attributed to both crude approximation in deriving the asymptotic formula (3) and the slow convergence of the function atan to $\frac{\pi}{2}$. This is in stark contrast with the classical case $\alpha=2$, where the asymptotic formula is very accurate even for relatively small $n$ [4]. The first ten eigenvalues are shown in Figure 2 for $\alpha=1.10$ and 1.75. We observe that, at least for $\alpha$ not close to unity, the asymptotic formula (3) is a poor approximation for small spectral numbers. So one cannot make much use of this from finite spectral data consisting of the lowest frequencies and it is precisely such data that is most easily measured in practice. 

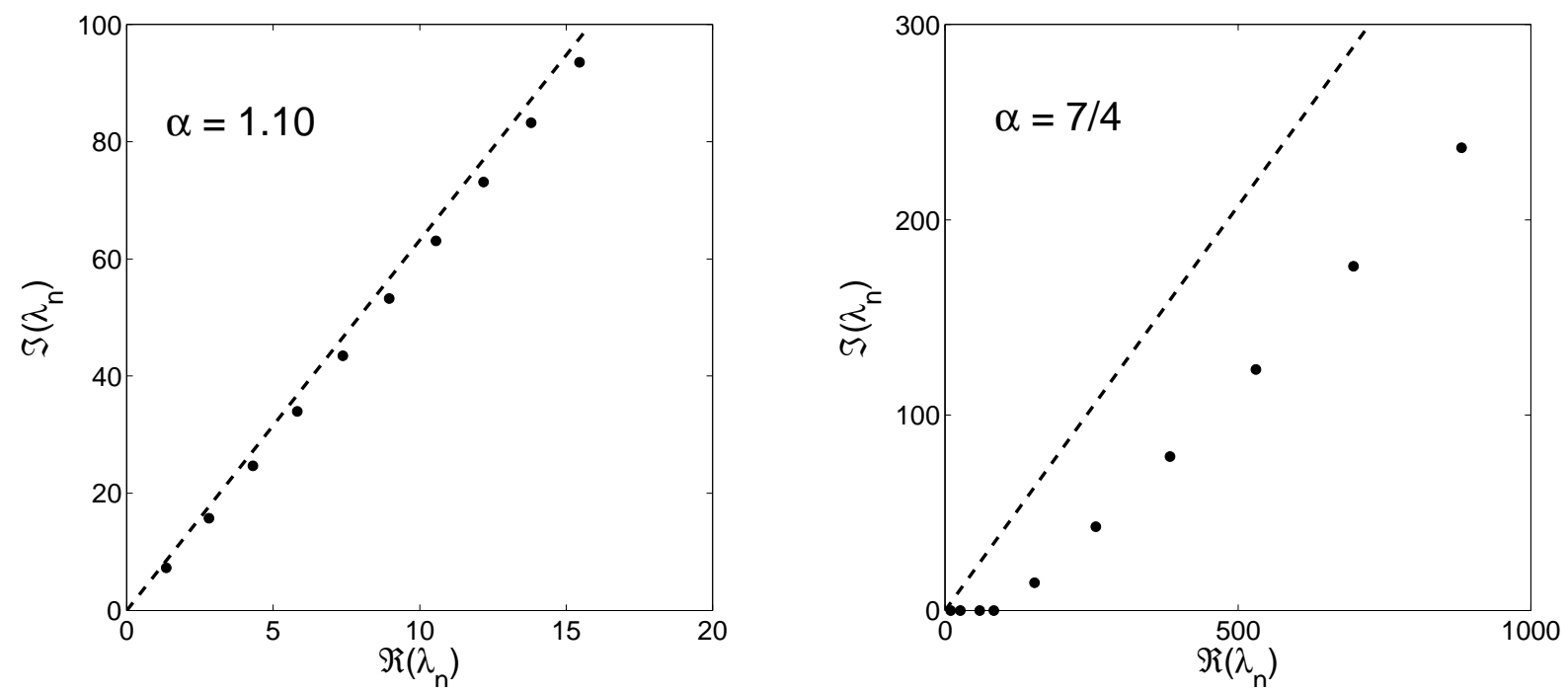

Figure 2: The first ten eigenvalues for $\alpha=1.10$ (left) and $\alpha=1.75$ (right). Here the dashed line represents the asymptotic with angle $\left(1-\frac{\alpha}{2}\right) \pi$.

The eigenfunctions $u_{n}$ for the operator $-D_{0}^{\alpha}$ are given by $u_{n}=x E_{\alpha, 2}\left(-\lambda_{n} x^{\alpha}\right)$. These functions are reminiscent of sinusoids (cf. Figure 3), the eigenfunctions for $\alpha=2$, but significantly attenuated close to $x=1$. The degree of attenuation strongly depends on fractional order $\alpha$. It is observed that for a complex eigenvalue, the number of interior zeros of the real and imaginary parts of the respective eigenfunction $u_{n}$ always differs by one, and that (either real or imaginary part) for two consecutive eigenvalues differs by two. However, the number of zeros for the consecutive real eigenvalues differ only by one (see the last row of Figure 3) just as for the classical SLP.

In the classical inverse SLP almost all reconstruction algorithms make use, directly or indirectly, of the fact that the eigenvalues are simple and, moreover, for potentials adjusted to have mean zero, there is an interval condition: there is exactly one (Dirichlet) eigenvalue in each interval $(n \pi,(n+1) \pi)[4$, Chap. 3]. In fact a similar result can be shown for the function $E_{\alpha, \beta}$ with $\alpha=2$ and $1<\beta<3$ [8, Thm. 1.4-2]. However, if $1<\alpha<2$ then this extremely useful property is either no longer ensured, or indeed does not hold. The asymptotic formula shows that all sufficiently large eigenvalues are simple, but it remains an open question if this is true in general - even for the case $q=0$ !

In the classical case, the eigenfunctions have the asymptotic form $u_{n}(x)=\sin \left(\sqrt{\lambda_{n}} x\right) / \sqrt{\lambda_{n}}\left(\cos \left(\sqrt{\lambda_{n}} x\right)\right.$ in the case of non-Dirichlet conditions at $x=0)$ [4], and these are very good approximations for moderately sized smooth potentials. Thus distinguishing eigenfunctions as well as eigenvalues is straightforward. Indeed, this clear correspondence also carries over to the potential recovery: if we are missing information about the $m^{\text {th }}$ eigenvalue, then we are unable to obtain information about the $m^{\text {th }}$ Fourier mode of the potential [9] but the other modes are essentially unaffected.

In the case $\alpha<2$, the picture is quite different. It is known that for $\alpha>5 / 3$ there are at least two real zeros (and hence eigenvalues) to the function $E_{\alpha, 2}(z)$ [20, Thm. 1]. Careful numerical experiments indicate that the first real zeros appear around $\alpha=1.6$ (more precisely within the interval $(1.5991152,1.5991153))$ and they occur in pairs (there are 4 real eigenvalues for $\alpha=1.75$ as Figure 2 shows) so there appears to be always an even number of these. However these pairs can be quite close to each other and so there is no possible interval condition. For example, with $\alpha=1.5991153$, the two smallest eigenvalues in magnitude are real with approximate values 14.0024 and 14.0150. Further refinement indicates these two eigenvalues are genuinely simple. The eigenfunction of the lower eigenvalue has no zeros in $(0,1)$, while the larger has one single zero and so they are linearly independent. However, the zero occurs at $x \approx 0.9994$ and the supremum norm difference of the two is less than $1.834 \times 10^{-4}$. Thus for all 

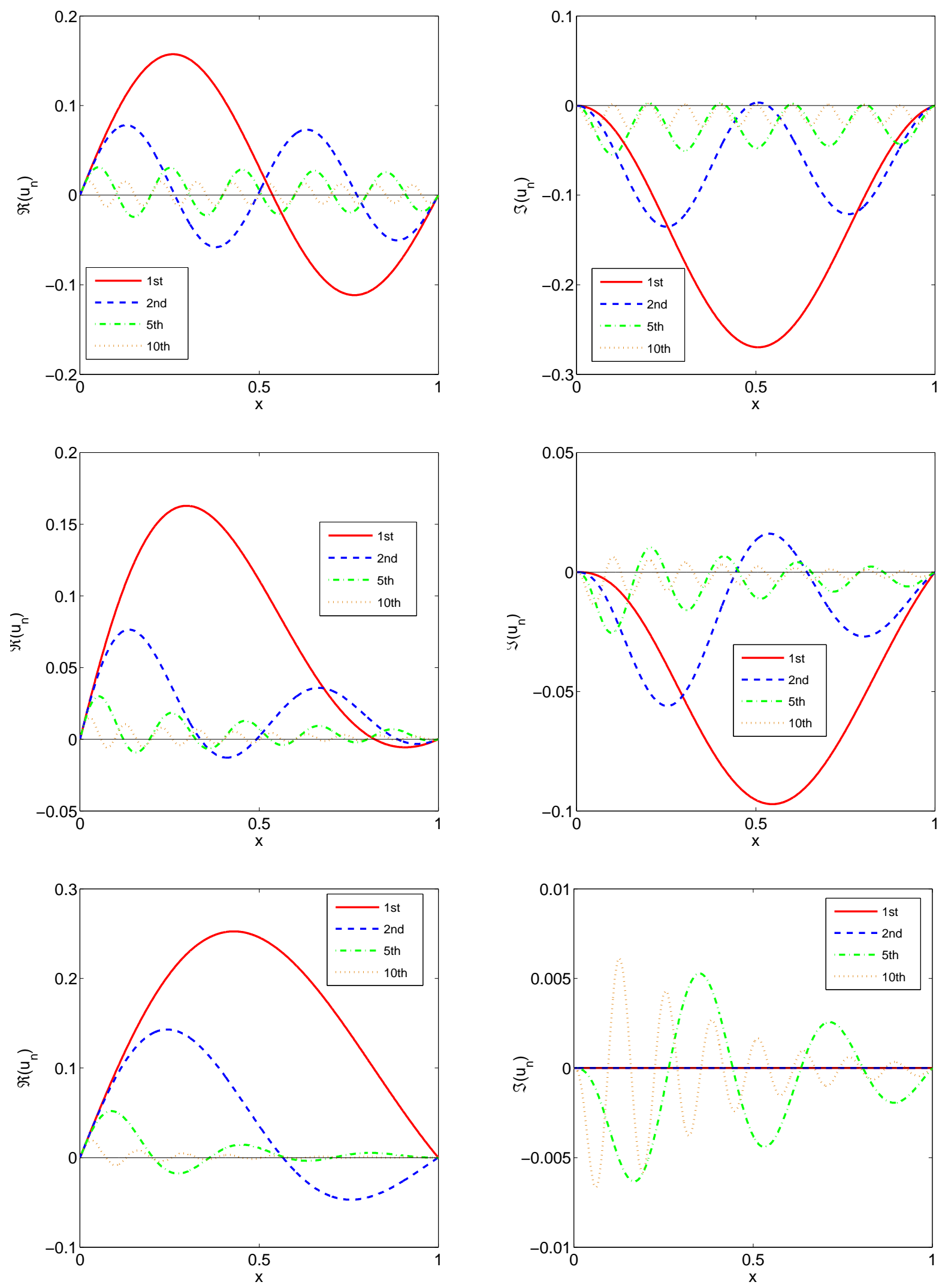

Figure 3: Real (left) and imaginary (right) parts of eigenfunctions $u_{n}$ for $\alpha=1.10,1.50$ and 1.75 (from top to bottom). 
practical computational purposes, neglecting one of these pairs will have no effect. With $\alpha=1.599025$, there are no real roots and the two smallest eigenvalues in magnitude are the complex conjugate pairs $14.0062 \pm 0.1955 \mathrm{i}$. As we noted above, the imaginary part of the corresponding eigenfunction has no zeros in $(0,1)$, and the real part does indeed have a zero in this interval. However, this is at a point $x_{0} \in(0.9997,0.9998)$ and the maximum value of the real part on $\left(x_{0}, 1\right)$ is less that $5.2501 \times 10^{-9}$. Thus practically speaking, this zero plays no computational role. These examples also highlight the difficulty in obtaining analytic results or estimates on the properties of the eigenvalues or eigenfunctions.

\subsection{Differential operator $-D_{0}^{\alpha}+q(x)$}

In the classical SLP, the presence of the potential $q(x)$ influences the eigenvalues by

$$
\lambda_{n}(q)=\lambda_{n}(0)+\int_{0}^{1} q(t) d t+c_{n},
$$

where the remainders $c_{n}$ decay to zero as $n \rightarrow+\infty$. The sequence $\left\{c_{n}\right\}$ effectively encodes all the information about the potential $q(x)$. This formula represents only a qualitative behavior. In practice, the decay rate of $c_{n}$ can differ markedly, dependent on the smoothness of the potential: the smoother is the potential, the faster is the decay. Numerically, we can observe similar behavior for fractional SLPs.
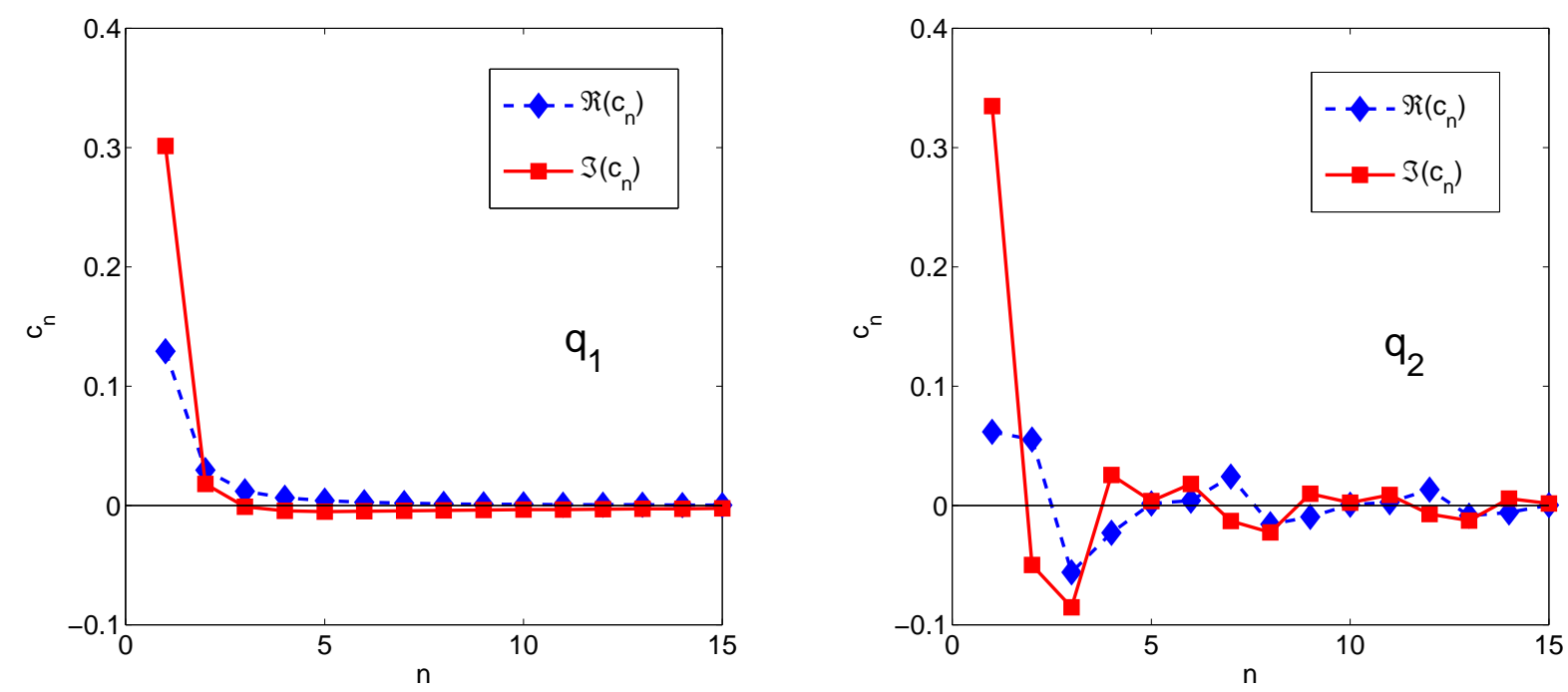

Figure 4: The decay of the remainder $c_{n}$ for $\alpha=3 / 2$.

To illustrate the point, we compute the eigenvalues for the following two potentials

$$
q_{1}(x)=20 x^{3}\left(e^{-\left(x-\frac{1}{2}\right)^{2}}-e^{-\frac{1}{4}}\right) \quad \text { and } \quad q_{2}(x)= \begin{cases}-2 x, & x \in\left[0, \frac{1}{5}\right] \\ -\frac{4}{5}+2 x, & x \in\left[\frac{1}{5}, \frac{2}{5}\right] \\ 1, & x \in\left[\frac{3}{5}, \frac{4}{5}\right] \\ 0, & \text { elsewhere }\end{cases}
$$

The profiles of the potentials can be found in Figure 5 shown later in $\S 3.2$. The differences between the eigenvalues for the differential operator $-D_{0}^{\alpha}+q(x)$ and that of $-D_{0}^{\alpha}+\int_{0}^{1} q(t) d t$ are shown in Figure 4 for $\alpha=3 / 2$. The decay of the remainders $c_{n}$ is much faster for the smooth potential $q_{1}$, which holds for both real and imaginary parts of eigenvalues. Hence, the first few eigenvalues might allow a reasonable reconstruction of the potential in the inversion procedures. In contrast, in case of the discontinuous potential $q_{2}$, the remainder $c_{n}$ still has noticeably large oscillations for $n$ up to 15 . These observations 
are also valid for other $\alpha$ values, and concur with that for the classical SLP [4, Figure 1, pp. 82]. We note that the presence of a nonzero potential may render two neighboring real eigenvalues into a pair of complex eigenvalues. For example, in case of $\alpha=1.6$, there are two real eigenvalues for $q=0$, i.e., 13.4205 and 14.6454, whereas that for the potentials $q_{1}$ and $q_{2}$ are given by the complex conjugate pair $14.6843 \pm 1.7197 \mathrm{i}$ and $14.2242 \pm 1.7910 \mathrm{i}$, respectively.

\section{An inverse Sturm-Liouville problem}

Now we turn to the inverse problem of recovering the potential $q(x)$ in the fractional SLP (1) from (finite) spectral data $\left\{\lambda_{n}\right\}_{n=1}^{N}$, with the help of a simplified Newton method. Numerically we observe that one single spectrum can uniquely determine a general potential.

\subsection{A Newton method}

We shall numerically solve the inverse SLP by a Newton type method based on that of [13] for the classical case. First, we denote by $u(q, \lambda)$ the solution to the initial value problem

$$
\left\{\begin{array}{l}
-D_{0}^{\alpha} u+q u=\lambda u \quad 0<x<1 \\
u(0)=0, \quad u^{\prime}(0)=1
\end{array}\right.
$$

Obviously, the number $\lambda \in \mathbb{C}$ will be an eigenvalue if the solution $u(q, \lambda)$ satisfies $u(q, \lambda)(1)=0$.

The knowledge of only finite spectral data $\left\{\lambda_{n}\right\}_{n=1}^{N}$ or equivalently the vector $\Lambda_{N}=\left(\lambda_{1}, \ldots, \lambda_{N}\right)^{\mathrm{t}} \in$ $\mathbb{C}^{N}$, as is often the case in practice, precludes the determination of an arbitrary potential $q$. Instead, we seek a potential in a finite-dimensional space spanned by the basis $\left\{w_{k}\right\}$, and we take its dimension to be $M \leq N$, allowing us the possibility to reduce this value for purposes of regularization, i.e.,

$$
q^{M}(x)=\sum_{k=1}^{M} q_{k} w_{k}(x) .
$$

Now the problem is to find $q^{M} \in \operatorname{span}\left(\left\{w_{k}\right\}\right)$ such that $u\left(q^{M}, \lambda\right)(1)$ vanishes, i.e., $u$ satisfies also the desired right-side boundary condition. We define a nonlinear mapping $F: \mathbb{R}^{M} \mapsto \mathbb{C}^{N}$ by

$$
F_{n}\left(\Lambda_{N}, q^{M}\right)=u\left(q^{M}, \lambda_{n}\right)(1) \quad n=1, \ldots, N
$$

For the given set $\Lambda_{N}$ of eigenvalues, we attempt to solve the system of nonlinear equations

$$
F\left(\Lambda_{N}, q^{M}\right)=0
$$

Thus Newton's method emerges as a natural candidate (see [13] for the case $\alpha=2$ ) and we can proceed as follows: given an initial guess $q^{0} \in \operatorname{span}\left(\left\{w_{k}\right\}_{k=1}^{M}\right)$, we repeat the iteration

$$
\mathbf{q}^{n+1}=\mathbf{q}^{n}-\left(F^{\prime}\left(\Lambda_{N}, q^{n}\right)\right)^{-1}\left(F\left(\Lambda_{N}, q^{n}\right)\right) \quad n=0,1, \ldots
$$

where the vector $\mathbf{q}^{n}=\left(q_{1}^{n}, \ldots, q_{M}^{n}\right) \in \mathbb{R}^{M}$ refers to the expansion coefficients of the approximate potential $q^{n}$ in the basis $\left\{w_{k}\right\}$, until a certain stopping criterion is reached. The entries of the $\operatorname{Jacobian} F^{\prime}\left(\Lambda_{N}, q\right)$ can be found by solving a set of fractional differential equations as we show in the next lemma.

Lemma 3.1. The $n k^{t h}$ entry of the Jacobian $F^{\prime}\left(\Lambda_{N}, q\right)$, i.e., $\frac{\partial F_{n}\left(\Lambda_{N}, q\right)}{\partial q_{k}}$, is given by $v\left(\lambda_{n}, q, w_{k}\right)(1)$, where the function $v\left(\lambda_{n}, q, w_{k}\right)$ satisfies

$$
\left\{\begin{array}{l}
-D_{0}^{\alpha} v+q v=\lambda_{n} v-w_{k} u\left(\lambda_{n}, q\right) \quad 0<x<1 \\
v(0)=0, \quad v^{\prime}(0)=0
\end{array}\right.
$$


Therefore, evaluating either the map $F\left(\Lambda_{N}, q\right)$ or the Jacobian $F^{\prime}\left(\Lambda_{N}, q\right)$ incurs solving initial value problems of fractional order (cf. (5) and (7)), which can be numerically accomplished by the predictorcorrector method in Appendix A. The main computational effort in applying the Newton method (6) lies in computing the Jacobian $F^{\prime}\left(\Lambda_{N}, q\right)$. We note that for large $\lambda$ values, the initial value problem is very stiff, and a very refined mesh may be needed to resolve the problem to a prescribed accuracy.

Of particular interest is the special case $q=0$ and using the respective eigenvalues $\Lambda_{N, 0}$. Then the solution $v\left(\lambda_{n, 0}, 0, w_{k}\right)$ to problem (7) can be expressed in closed form using the Green's function of the operator $D_{0}^{\alpha}-\lambda[10$, eq. (4.1.74), p. 232]

$$
v\left(\lambda_{n, 0}, 0, w_{k}\right)(x)=\int_{0}^{x}(x-t)^{\alpha-1} E_{\alpha, \alpha}\left(-\lambda_{n, 0}(x-t)^{\alpha}\right) t E_{\alpha, 2}\left(-\lambda_{n, 0} t^{\alpha}\right) w_{k}(t) d t .
$$

With the approximation $F^{\prime}\left(\Lambda_{N, 0}, 0\right)$ in place of the Jacobian $F^{\prime}\left(\Lambda_{N}, q^{n}\right)$, the iteration (6) leads to the canonical frozen Newton's method, which is known to converge (locally) linearly. This reduces the computational expense enormously since computing the Jacobian $F^{\prime}\left(\Lambda_{N}, q^{n}\right)$ is online and very expensive, whereas $F^{\prime}\left(\Lambda_{N, 0}, 0\right)$ can be calculated offline. When solving (6), we stack the real and imaginary parts of the matrix $F^{\prime}\left(\Lambda_{N, 0}, 0\right)$ and the vector $F\left(\Lambda_{N}, q^{n}\right)$ as follows

$$
\mathbf{J}=\left[\begin{array}{c}
\Re\left(F^{\prime}\left(\Lambda_{N, 0}, 0\right)\right) \\
\Im\left(F^{\prime}\left(\Lambda_{N, 0}, 0\right)\right)
\end{array}\right] \quad \text { and } \quad \mathbf{r}^{n}=\left[\begin{array}{c}
\Re\left(F\left(\Lambda_{N}, q^{n}\right)\right) \\
\Im\left(F\left(\Lambda_{N}, q^{n}\right)\right)
\end{array}\right] .
$$

In this situation the system has real components only which ensures that the Newton update $\mathbf{J}^{-1} \mathbf{r}^{n}$ is always real. Experimentally, the frozen Newton's method converges fairly fast. As is a general rule for applying these type of methods to nonlinear inverse problem, the invertibility of the Jacobian is often very difficult to establish theoretically, and this is the case here. The latter obstacle is closely related to the lack of a uniqueness result for the inverse SLP problem. Consequently, we are not able to establish the convergence of the quasi-Newton scheme and, nor can we derive error estimates for the finite-dimensional approximations. However, it is conjectured that $F^{\prime}\left(\Lambda_{N, 0}, 0\right)$ for any $\alpha \in(1,2)$ is invertible in view of our experimental experiences.

\subsection{Numerical results and discussions}

Now we are ready to present numerical reconstructions of the potentials $q_{1}$ and $q_{2}$ from finite spectral data $\Lambda_{N}$. For the spectral data $\left(\Lambda_{N}\right)$ generation and for the inversion step, we solve the initial value problem (5) with the predictor-corrector algorithm (see Appendix A) with a mesh size $h=1 \mathrm{e}-3$ and $h=1.25 \mathrm{e}-3$, respectively, in order to reduce the most obvious form of "inverse crime", but not completely due to the use of identical predictor-corrector algorithm in the forward and inverse steps. The eigenvalues are found by a quasi-Newton method in Appendix B. The basis set $\left\{w_{k}\right\}$ is fixed at $\{\sin k \pi\}$. All the computations were performed with MATLAB R. 7.12.0(2011a) on a dual core desktop computer.

Table 1: Reconstruction error $e$ for the potentials.

\begin{tabular}{|c|ccccccccc|}
\hline$\alpha$ & 1.02 & 1.1 & $6 / 5$ & $4 / 3$ & $3 / 2$ & $5 / 3$ & $7 / 4$ & $9 / 5$ & 1.85 \\
\hline$q_{1}(N=8)$ & $4.217 \mathrm{e}-3$ & $4.156 \mathrm{e}-3$ & $4.071 \mathrm{e}-3$ & $4.013 \mathrm{e}-3$ & $4.042 \mathrm{e}-3$ & $4.690 \mathrm{e}-3$ & $9.169 \mathrm{e}-3$ & $2.576 \mathrm{e}-2$ & $1.218 \mathrm{e}-1$ \\
$q_{2}(N=10)$ & $1.983 \mathrm{e}-1$ & $1.983 \mathrm{e}-1$ & $1.983 \mathrm{e}-1$ & $1.984 \mathrm{e}-1$ & $1.988 \mathrm{e}-1$ & $2.026 \mathrm{e}-1$ & $3.331 \mathrm{e}-1$ & $1.709 \mathrm{e} 0$ & $3.469 \mathrm{e} 0$ \\
\hline
\end{tabular}

The numerical results are summarized in Table 1 and Figure 5. In the table, the error $e$ of an approximation $\tilde{q}$ to the potential $q$ is defined by $e=\|q-\tilde{q}\|_{L^{2}(0,1)}$. We have experimented the reconstruction algorithm with different numbers of basis and eigenvalues. Although the concrete numbers differ slightly, the observations drawn from these results are the same. Hence, we shall present only the results with $N=M=8$ and $N=M=10$ for the potentials $q_{1}$ and $q_{2}$, respectively. We remark that the convergence of the frozen Newton method is steady and fast, effective numerical convergence being generally achieved within five iterations, cf. Figure 6 for an illustration. 

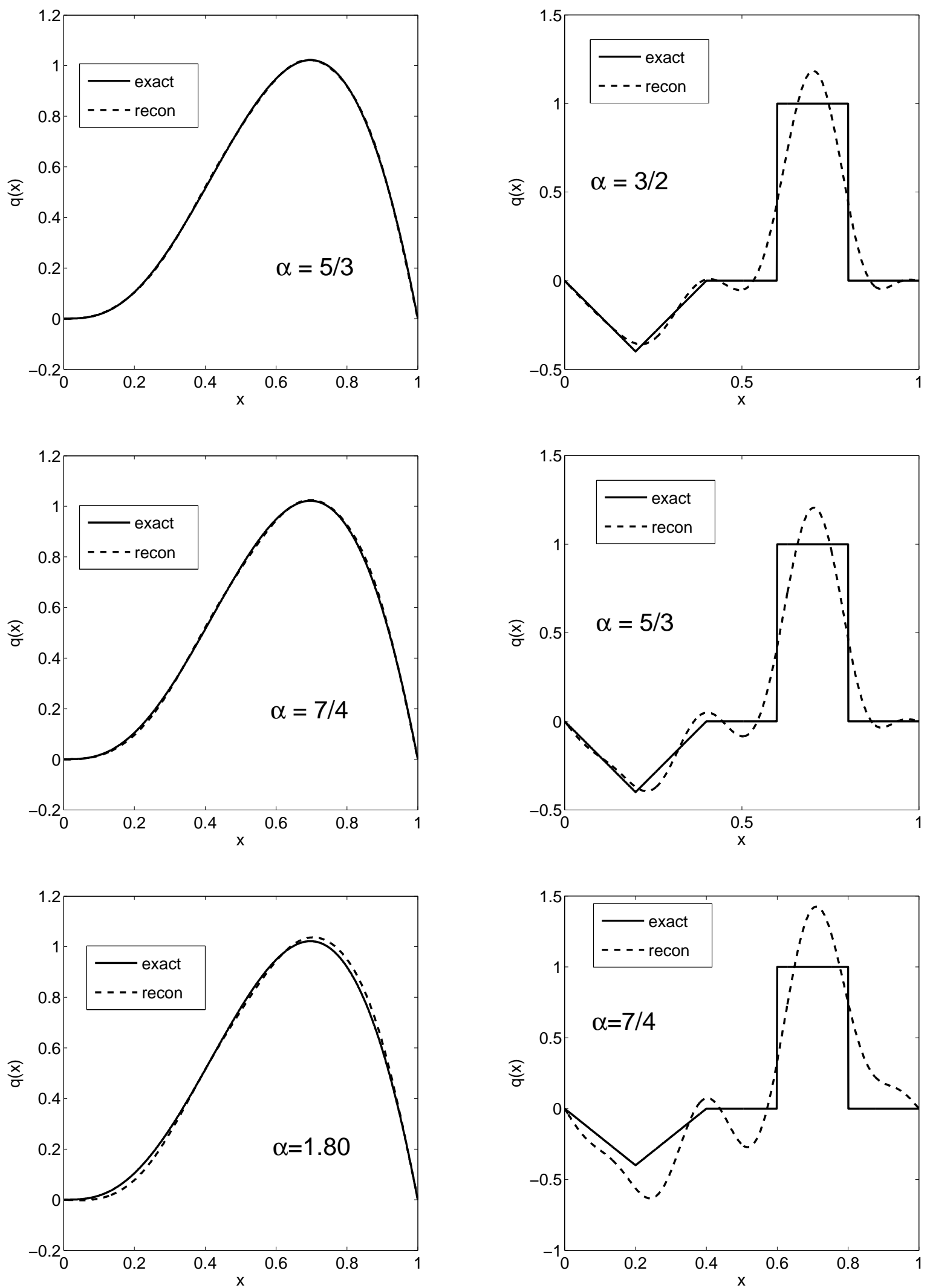

Figure 5: Numerical reconstructions for the potentials $q_{1}$ (left) and $q_{2}$ (right). 
Table 2: Condition number of the stacked Jacobian matrix $\mathbf{J}$.

\begin{tabular}{|c|ccccccccc|}
\hline \multicolumn{1}{|c|}{$\alpha$} & 1.02 & 1.1 & $6 / 5$ & $4 / 3$ & $3 / 2$ & $5 / 3$ & $7 / 4$ & $9 / 5$ & 1.85 \\
\hline$N=M=5$ & $5.313 \mathrm{e} 0$ & $6.393 \mathrm{e} 0$ & $8.302 \mathrm{e} 0$ & $1.236 \mathrm{e} 1$ & $2.095 \mathrm{e} 1$ & $2.716 \mathrm{e} 2$ & $2.573 \mathrm{e} 3$ & $4.136 \mathrm{e} 3$ & $1.193 \mathrm{e} 4$ \\
$N=M=8$ & $8.267 \mathrm{e} 0$ & $1.054 \mathrm{e} 1$ & $1.479 \mathrm{e} 1$ & $2.439 \mathrm{e} 1$ & $4.608 \mathrm{e} 1$ & $6.109 \mathrm{e} 2$ & $8.767 \mathrm{e} 3$ & $3.397 \mathrm{e} 5$ & $1.114 \mathrm{e} 5$ \\
$N=M=10$ & $1.049 \mathrm{e} 1$ & $1.399 \mathrm{e} 1$ & $2.082 \mathrm{e} 1$ & $3.743 \mathrm{e} 1$ & $7.978 \mathrm{e} 1$ & $1.043 \mathrm{e} 3$ & $1.110 \mathrm{e} 4$ & $3.298 \mathrm{e} 5$ & $3.711 \mathrm{e} 5$ \\
$N=M=15$ & $1.656 \mathrm{e} 1$ & $2.411 \mathrm{e} 1$ & $4.037 \mathrm{e} 1$ & $8.586 \mathrm{e} 1$ & $2.299 \mathrm{e} 2$ & $3.998 \mathrm{e} 3$ & $8.524 \mathrm{e} 4$ & $1.345 \mathrm{e} 6$ & $1.895 \mathrm{e} 7$ \\
\hline
\end{tabular}

For $\alpha$ in the range $\left(1, \frac{5}{3}\right]$, the error $e$ of the recovered potential $q_{1}$ largely stays constant, and then it deteriorates as the exponent $\alpha$ gets closer to 2 . Some reconstructed profiles are shown in the left column of Figure 5. The reconstructions are in excellent agreement with the true potential for $\alpha \in\left(1, \frac{5}{3}\right]$. Hence, one single spectrum allows accurately reconstructing a general potential. For $\alpha$ value very close to 2 , e.g., $\alpha=1.9$, we are unable to obtain a good approximation by even using 15 eigenvalues. This is expected since in the limit case $\alpha=2$, it is well known that one single spectrum is insufficient for completely determine the potential, and only the symmetric part can be recovered [13]. This is corroborated by the dramatic increase of the condition number of the Jacobian $F^{\prime}\left(\Lambda_{N, 0}, 0\right)$, cf. Table 2: the conditioning of the stacked Jacobian $\mathbf{J}$ worsens steadily as the exponent $\alpha$ approaches 2 as expected since it must be singular for $\alpha=2$. The ill-conditioning is inherently related to lack of information and is particularly relevant for noisy data. There are several possible remedies. The most straightforward one is to use a smaller number of basis functions which directly leads to a better conditioned Jacobian matrix. This strategy has proved very effective in our experiments. Another very natural approach would be to employ regularization techniques, e.g., Tikhonov regularization and truncated singular value decomposition. Perhaps surprisingly, these were not able to produce better results and sometimes resulted in inferior ones.

Similar observations can be drawn from the numerical results for the discontinuous potential $q_{2}$; see the right column of Figure 5. The reconstruction in case of $\alpha=\frac{3}{2}$ (or in fact, any lesser value of $\alpha$ ) is very close to the best Fourier approximation of the exact potential, and given our basis representation approach, it represents an optimal reconstruction. For larger $\alpha$, and strongly so as $\alpha$ approaches $\alpha=2$, the higher-order Fourier coefficients needed for faithfully capturing the potential $q_{2}$ are less well resolved and hence the reconstructions are less accurate. In comparison with the smooth potential, the onset of deterioration also occurs for a smaller $\alpha$ value.

These empirical observations strongly indicate that the eigenvalues of the fractional SLP contain more information than that of the standard second-order SLP, especially for $\alpha$ values sufficiently away from 2. This might be attributed to the fact that the eigenvalues of the fractional SLP are genuinely complex, rather than real as for the classical SLP. Similar phenomena have been observed previously in related problems in second-order SLPs and first-order systems. In [23] the authors demonstrated the unique identifiability of the potential from one complex eigenvalue sequence for the second-order SLP with a complex impedance boundary condition. Yamamoto [30] showed that two sequences of (complex) eigenvalues of a certain first-order matrix operator uniquely determine two unknown coefficients in the operator.

Lastly, we contrast the experimentally observed uniqueness for the fractional SLP with the classical $\alpha=2$ in the linearized case. It is tempting to attribute the very different behavior of the fractional case $1<\alpha<2$ from the classical $\alpha=2$ to the "anomalous diffusion" assumption. But the behavior for $\alpha=2$ might be viewed as the unusual circumstance. To this end, we let $q_{1}$ and $q_{2}$ be two potentials, and $\left\{\lambda_{n}\right\}$ and $\left\{\mu_{n}\right\}$ be respective Dirichlet spectrum, and $\left\{u_{n}\right\}$ and $\left\{v_{n}\right\}$ eigenfunctions. Then

$$
\int_{0}^{1}\left(q_{1}-q_{2}\right) u_{n} v_{n} d x=\left(\lambda_{n}-\mu_{n}\right) \int_{0}^{1} u_{n} v_{n} d x
$$

If the potentials have the same spectrum, then

$$
\int_{0}^{1}\left(q_{1}-q_{2}\right) u_{n} v_{n} d x=0 \quad \forall n
$$



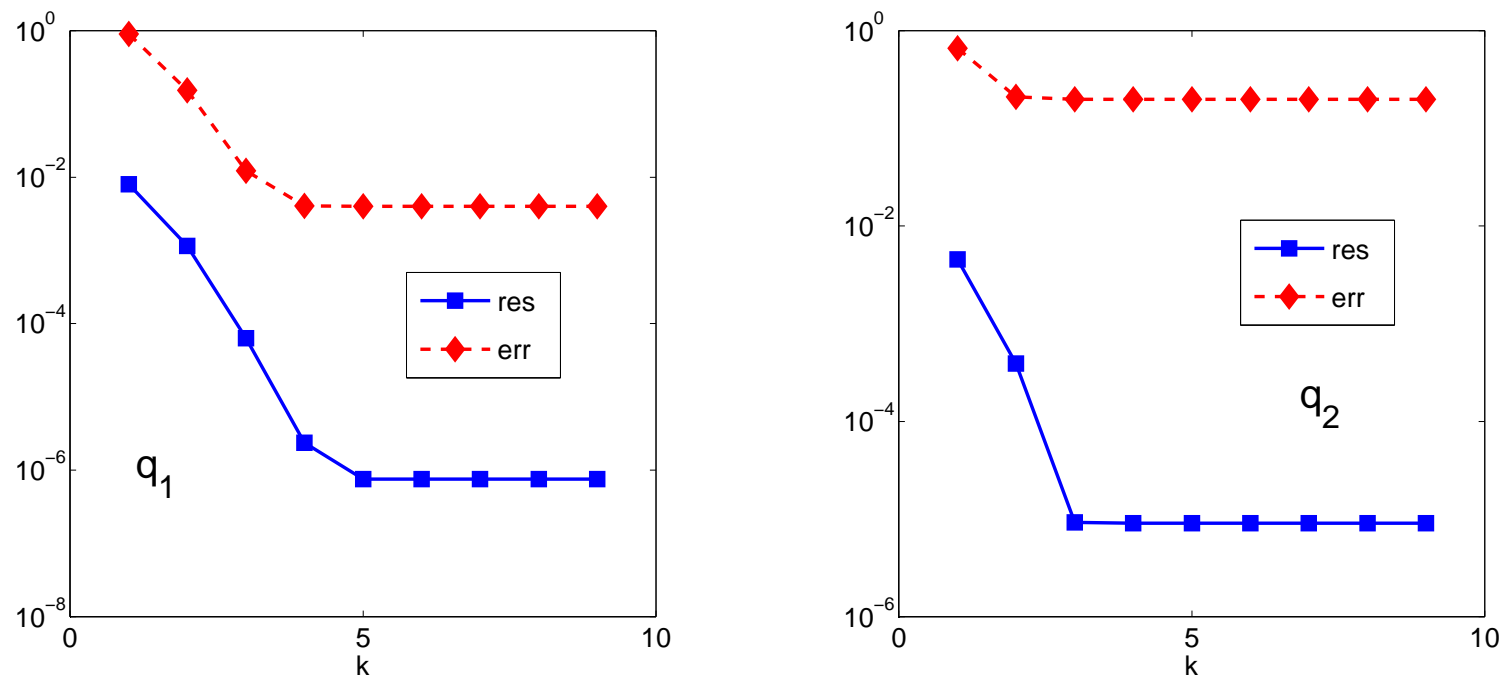

Figure 6: Convergence of the frozen Newton method for $\alpha=3 / 2$. Here res and err refer to the residual $\left\|F\left(q, \Lambda_{N}\right)\right\|$ and the error $e$, respectively.

Now in the case $\alpha=2$, the eigenfunctions $\left\{u_{n}\right\}$ behave asymptotically like $\frac{1}{\sqrt{\lambda_{n}}} \sin \left(\sqrt{\lambda_{n}} x\right)+O\left(\frac{1}{\sqrt{\lambda_{n}}}\right)$ and $\sqrt{\lambda_{n}}=n \pi+O\left(\frac{1}{n}\right)$, and similarly $\left\{v_{n}\right\}$ and $\mu_{n}$ [4, Chap. 3]. Meanwhile, the asymptotic expansion implies that the mean values of the potentials $q_{1}$ and $q_{2}$ are identical. Hence, the identity $\sin ^{2} n \pi x=$ $\frac{1}{2}(1-\cos 2 n \pi x)$ implies that to leading order, there holds

$$
\int_{0}^{1}\left(q_{1}-q_{2}\right) \cos (2 n \pi x) d x=0 \quad \forall n .
$$

Therefore, to leading order, the even part of the potential difference $q_{1}-q_{2}$ is zero; that is the Dirichlet spectrum determines the even part of the difference, but equally, gives no information on the odd part. A refinement of this idea leads to the original uniqueness proof due to Borg [1] of both the two spectrum problem and the case when the potential is known to be symmetric.

However, this proof and its consequences rely on the fact that the set of squares of the eigenfunctions $\left\{u_{n}\right\}$ only spans effectively "one half" of the space $L^{2}(0,1)$, upon ignoring the small errors in the procedure, and this is due entirely to the trigonometric identity relating the squares of eigenfunctions of order $n$ to eigenfunctions of order $2 n$. In the case $1<\alpha<2$, even for $q=0$, we have no such identity relating $u_{n}^{2}$ to another single eigenfunction; in fact we would expect an expression of the form $u_{n}^{2}(x)=\sum_{k=1}^{\infty} a_{k} u_{k}(x)$ where none of the complex-valued sequence $\left\{a_{k}\right\}$ vanishes. Looked at in this light, the anomalous behavior is in fact exhibited by the case $\alpha=2$.

\section{Concluding remarks}

We have presented a first numerical study of the forward and inverse Sturm-Liouville problems for fractional differential operators. The numerical results indicate a stunning observation: one single spectrum can uniquely determine a general potential, and a few (finite) spectral data allow very good reconstructions, provided that the fractional order $\alpha \in(1,2)$ is sufficiently less than 2 .

These experimental observations lead to a number of interesting conjectures for the fractional SturmLiouville problem:

(a) All the eigenvalues of the operator $-D_{0}^{\alpha}(\alpha \in(1,2))$ are simple. 
(b) The (Dirichlet) eigenvalues of $-D_{0}^{\alpha}+q(x)(1<\alpha<2)$ obey the relation (4) with $\left\{c_{n}\right\} \in \ell^{2}$.

(c) For any $\alpha \in(1,2)$, one single (Dirichlet) spectrum completely determines the potential $q$ in the fractional Sturm-Liouville problem (1).

(d) While (c) is the optimal result, a weaker version might be more tractable: to show the injectivity of the linearized map.

(e) There are clear extensions to other boundary conditions and also the case of the half line.

Apart from these theoretical questions, the rigorous analysis of relevant numerical schemes is also of immense interest. These include error estimates for the eigenvalue approximations, convergence and convergence rates of the quasi-Newton scheme etc. A better understanding of the analytical aspects of the fractional Sturm-Liouville problem is crucial for addressing these numerical issues.

\section{Acknowledgements}

This work is supported by Award No. KUS-C1-016-04, made by King Abdullah University of Science and Technology (KAUST), and NSF award DMS-0715060.

\section{A Predictor-corrector algorithm}

Here we describe a predictor-corrector algorithm for fractional initial value problems based on the idea in the work [6]. There are several errors in the formulas in [6], in, e.g. eq. (15). Hence we present necessary details here. Consider the initial value problem (with $\alpha \in(1,2)$ )

$$
\left\{\begin{array}{l}
D_{0}^{\alpha} u=f(x, u(x)) \quad 0<x<1, \\
u(0)=u_{0}, \quad u^{\prime}(0)=u_{0}^{(1)} .
\end{array}\right.
$$

This initial value problem is equivalent to the Volterra integral equation [10, eq. (3.1.41), pp. 141]

$$
u(x)=u_{0}+u_{0}^{(1)} x+\frac{1}{\Gamma(\alpha)} \int_{0}^{x}(x-t)^{\alpha-1} f(t, u(t)) d t .
$$

To solve equation (8), we partition the unit interval $[0,1]$ into a uniform mesh $\mathcal{T}=\left\{x_{k}=k h, k=\right.$ $0,1, \ldots, K\}, K \in \mathbb{N}$, with a mesh size $h=\frac{1}{K}$. The predictor-corrector algorithm in [6] extends the Adams-Bashforth-Moulton method. The predictor step applies the left rectangle rule (with the kernel $\left(x_{k+1}-x\right)^{\alpha-1}$ being the weight) to the integral in (8), i.e.,

$$
\int_{0}^{x_{k+1}}\left(x_{k+1}-x\right)^{\alpha-1} g(x) d x \approx \frac{h^{\alpha}}{\alpha} \sum_{j=0}^{k} b_{j, k+1} g\left(x_{j}\right),
$$

where the integrand $g$ is continuous and the weights $b_{j, k+1}$ are given by

$$
b_{j, k+1}=(k+1-j)^{\alpha}-(k-j)^{\alpha}, \quad j=0,1, \ldots, k .
$$

The corrector step applies the trapezoidal rule (again with the kernel $\left(x_{k+1}-x\right)^{\alpha-1}$ being the weight) to the integral in (8), i.e.,

$$
\int_{0}^{x_{k+1}}\left(x_{k+1}-x\right)^{\alpha-1} g(x) d x \approx \frac{h^{\alpha}}{(\alpha+1) \alpha} \sum_{j=0}^{k+1} a_{j, k+1} g\left(x_{j}\right),
$$


where the weights $a_{j, k+1}$ are given by

$$
a_{j, k+1}= \begin{cases}k^{\alpha+1}-(k-\alpha)(k+1)^{\alpha}, & \text { if } j=0, \\ (k-j+2)^{\alpha+1}+(k-j)^{\alpha+1}-2(k-j+1)^{\alpha+1}, & \text { if } j=1, \ldots, k, \\ 1, & \text { if } j=k+1 .\end{cases}
$$

The predictor-corrector algorithm applies repeatedly the above two quadrature rules to get the approximations $u_{h}^{\mathrm{p}}\left(x_{k+1}\right)$ and $u_{h}^{\mathrm{c}}\left(x_{k+1}\right)$ respectively by

$$
\begin{aligned}
& u_{h}^{\mathrm{p}}\left(x_{k+1}\right)=u_{0}+u_{0}^{(1)} x_{k+1}+\frac{h^{\alpha}}{\Gamma(1+\alpha)} \sum_{j=0}^{k} b_{j, k+1} f\left(x_{j}, u_{h}\left(x_{j}\right)\right), \\
& u_{h}^{\mathrm{c}}\left(x_{k+1}\right)=u_{0}+u_{0}^{(1)} x_{k+1}+\frac{h^{\alpha}}{\Gamma(2+\alpha)} f\left(x_{k+1}, u_{h}^{\mathrm{p}}\left(x_{k+1}\right)+\frac{h^{\alpha}}{\Gamma(2+\alpha)} \sum_{j=0}^{k} a_{j, k+1} f\left(x_{j}, u_{h}\left(x_{j}\right)\right) .\right.
\end{aligned}
$$

Then the sought-for approximation $u_{h}\left(x_{k+1}\right)$ is obtained by re-evaluate the corrector step with $u_{h}^{\mathrm{c}}\left(x_{k+1}\right)$ in place of $u_{h}^{\mathrm{p}}\left(x_{k+1}\right)$. To further improve the accuracy of the approximation $\tilde{u}_{h}$, we adopt a Richardson extrapolation step, which reads $\tilde{u}_{h}=\frac{1}{3}\left(4 u_{\frac{h}{2}}-u_{h}\right)$. The solver for the initial value problem always incorporates this extrapolation step.

\section{B Quasi-Newton method for eigenvalue problem}

Next we describe a quasi-Newton method for finding an eigenvalue $\lambda$ to the SLP (1). The starting point of the method is the following obvious observation: any eigenvalue $\lambda$ to the SLP (1) is one root of the nonlinear map from the potential $q$ to $u(q, \lambda)(1)$, where $u(q, \lambda)$ is the solution to the initial value problem (5). Clearly, then the solution $u(q, \lambda)$ will also be the respective eigenfunction.

We shall find the eigenvalues to the SLP (1) by a quasi-Newton method, the secant method. The complete algorithm is listed in Algorithm 1. The stopping criterion at Step 8 can be based on monitoring the quasi-Newton update $\delta \lambda$ : if the increment $|\delta \lambda|$ falls below a given tolerance (which is set to $1.0 \times 10^{-12}$ in our computation), then the algorithm is terminated. Accurate initial guesses for the SLP (1) with zero potential can be obtained from the zeros of Mittag-Leffler function $E_{\alpha, 2}(-\lambda)$, which can be directly extracted by visualizing the function $E_{\alpha, 2}(-\lambda)$ [28]. That for a nonzero potential can be obtained by adding the integral $\int_{0}^{1} q(t) d t$ to the eigenvalues for zero potential. In practice, including a small imaginary part in the initial guesses is beneficial. Each iteration of the algorithm invokes solving one initial value problem, which is dominant in the expense. Our numerical experiences indicate that its convergence appears always superlinear, and the convergence basin is relatively large.

\section{References}

[1] G. Borg. Eine Umkehrung der Sturm-Liouvilleschen Eigenwertaufgabe. Acta Math., 76(1):1-96, 1946.

[2] J.-P. Bouchaud and A. Georges. Anomalous diffusion in disordered media: Statistical mechanisms, models and physical applications. Phys. Rep., 195(4-5):127-293, 1990.

[3] H. Brunner, L. Ling, and M. Yamamoto. Numerical simulations of 2D fractional subdiffusion problems. J. Comput. Phys., 229(18):6613-6622, 2010.

[4] K. Chadan, D. Colton, L. Päivärinta, and W. Rundell. An Introduction to Inverse Scattering and Inverse Spectral Problems. SIAM, Philadelphia, PA, 1997.

[5] J. Cheng, J. Nakagawa, M. Yamamoto, and T. Yamazaki. Uniqueness in an inverse problem for a one-dimensional fractional diffusion equation. Inverse Problems, 25(11):115002, 16, 2009. 


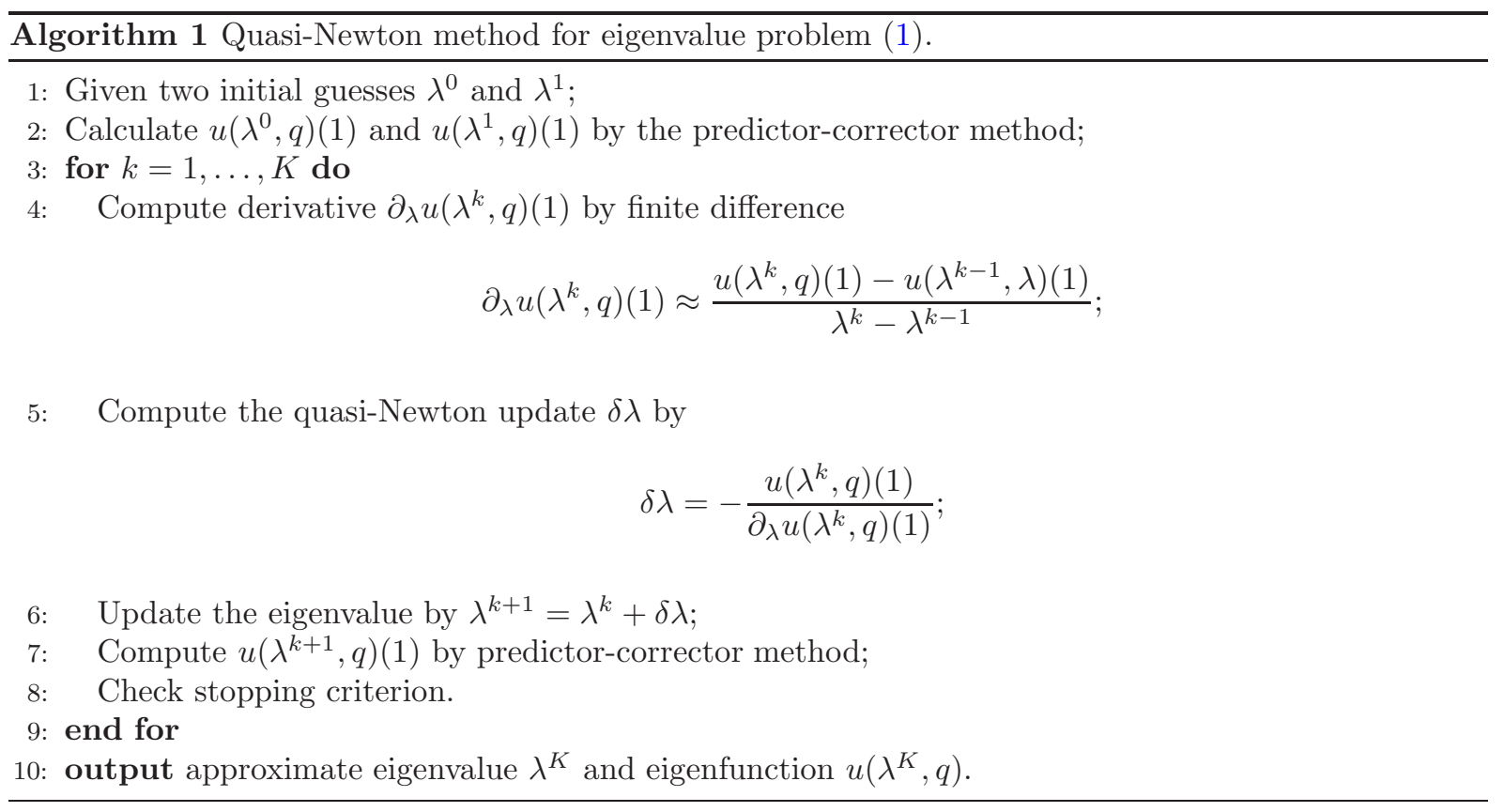

[6] K. Diethelm, N. J. Ford, and A. D. Freed. A predictor-corrector approach for the numerical solution of fractional differential equations. Nonlinear Dynam., 29(1-4):3-22, 2002.

[7] M. M. Djrbashian. A boundary value problem for a Sturm-Liouville type differential operator of fractional order. Izv. Akad. Nauk Armjan. SSR Ser. Mat., 5(2):71-96, 1970.

[8] M. M. Djrbashian. Harmonic Analysis and Boundary Value Problems in the Complex Domain. Birkhäuser Verlag, Basel, 1993.

[9] H. Hochstadt. The inverse Sturm-Liouville problem. Comm. Pure Appl. Math., 26:715-729, 1973.

[10] A. A. Kilbas, H. M. Srivastava, and J. J. Trujillo. Theory and Applications of Fractional Differential Equations. Elsevier, Amsterdam, 2006.

[11] Y. Lin and C. Xu. Finite difference/spectral approximations for the time-fractional diffusion equation. J. Comput. Phys., 225(2):1533-1552, 2007.

[12] J. J. Liu and M. Yamamoto. A backward problem for the time-fractional diffusion equation. Appl. Anal., 89(11):1769-1788, 2010.

[13] B. D. Lowe, M. Pilant, and W. Rundell. The recovery of potentials from finite spectral data. SIAM J. Math. Anal., 23(2):482-504, 1992.

[14] M. M. Meerschaert, D. A. Benson, and B. Bäumer. Multidimensional advection and fractional dispersion. Phys. Rev. E, 59(5):5026-5028, 1999.

[15] R. Metzler and J. Klafter. The random walk's guide to anomalous diffusion: a fractional dynamics approach. Phys. Rep., 339(1):1-77, 2000.

[16] R. Metzler and J. Klafter. The restaurant at the end of the random walk: recent developments in the description of anomalous transport by fractional dynamics. J. Phys. A: Math. Gen., 37(31):R161R208, 2004.

[17] R. Metzler and T. F. Nonnenmacher. Space- and time-fractional diffusion and wave equations, fractional Fokker-Planck equations, and physical motivations. Chem. Phys., 284(1-2):67-90, 2002. 
[18] A. M. Nahušev. The Sturm-Liouville problem for a second order ordinary differential equation with fractional derivatives in the lower terms. Dokl. Akad. Nauk SSSR, 234(2):308-311, 1977.

[19] I. Podlubny. Fractional Differential Equations. Academic Press, San Diego, CA, 1999.

[20] A. Y. Popov. On the number of real eigenvalues of a certain boundary-value problem for a secondorder equation with fractional derivative. J. Math. Sci., 151(1):2726-2740, 2008.

[21] N. Röhrl. A least-squares functional for solving inverse Sturm-Liouville problems. Inverse Problems, 21(6):2009-2017, 2005.

[22] Y. A. Rossikhin and M. V. Shitikova. Application of fractional calculus for dynamic problems of solid mechanics: novel trends and recent results. Appl. Mech. Rev., 63(1):010801, 52 pp., 2010.

[23] W. Rundell and P. Sacks. Numerical technique for the inverse resonance problem. J. Comput. Appl. Math., 170(2):337-347, 2004.

[24] W. Rundell and P. E. Sacks. Reconstruction techniques for classical inverse Sturm-Liouville problems. Math. Comp., 58(197):161-183, 1992.

[25] K. Sakamoto and M. Yamamoto. Initial value/boundary value problems for fractional diffusion-wave equations and applications to some inverse problems. J. Math. Anal. Appl., 382(1):426-447, 2011.

[26] W. R. Schneider and W. Wyss. Fractional diffusion and wave equations. J. Math. Phys., 30(1):134$144,1989$.

[27] A. M. Sedletskiı̌. Asymptotic formulas for zeros of functions of Mittag-Leffler type. Anal. Math., 20(2):117-132, 1994.

[28] H. Seybold and R. Hilfer. Numerical algorithm for calculating the generalized Mittag-Leffler function. SIAM J. Numer. Anal., 47(1):69-88, 2008/09.

[29] M. F. Shlesinger, B. J. West, and J. Klafter. Lévy dynamics of enhanced diffusion: Application to turbulence. Phys. Rev. Lett., 58(11):1100-1103, 1987.

[30] M. Yamamoto. Inverse spectral problem for systems of ordinary differential equations of first order. I. J. Fac. Sci. Univ. Tokyo Sect. IA Math., 35(3):519-546, 1988.

[31] G. H. Zheng and T. Wei. A new regularization method for the time fractional inverse advectiondispersion problem. SIAM J. Numer. Anal., 49(5):1972-1990, 2011. 